\title{
Severe Hydronephrosis in Nephrogenic Diabetes Insipidus
}

\author{
Hongbo Yang, Naishi Li, Lingling Xu and Weibo Xia
}

A

33-year-old woman was admitted to Peking Union Medical College hospital complaining of a 26-year history of polydipsia and polyuria. She had a family history of nephrogenic diabetes insipidus. Blood chemistry was remarkable for elevated sodium $>150 \mathrm{mmol} / \mathrm{L}$. A magnetic resonance imaging scan of the patient's brain revealed no abnormalities in the sella turcica. Water deprivation test demonstrated the presence of vasopressin resistant nephrogenic diabetes insipidus (NDI).

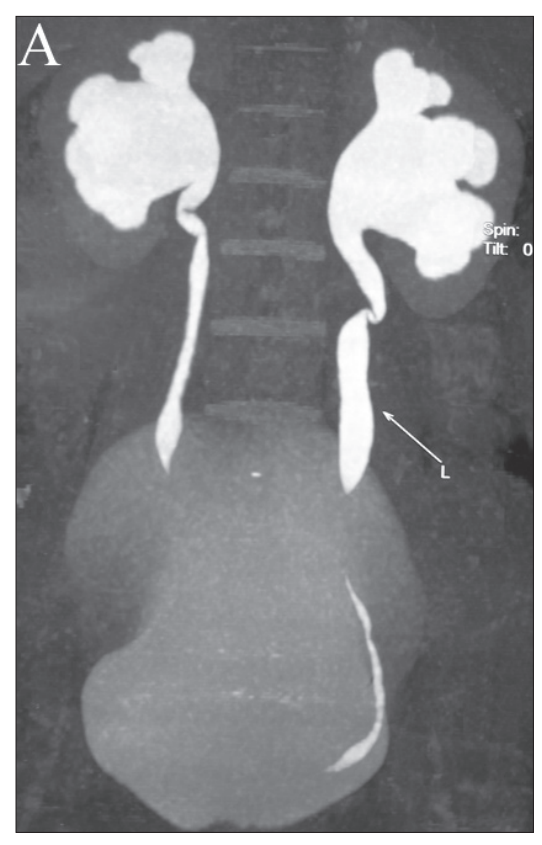

Figure 1A. Intravenous pyelography.
Massive bilateral hydronephrosis and megaureter were demonstrated by intravenous pyelography (figure 1A) and three-dimensional computed tomography (figure 1B) of the kidneys. The patient's total urine volume decreased by more than $50 \%$ after treatment with hydrochlorothiazide $25 \mathrm{mg}$ three times daily and spironolactone once daily. Hydronephrosis was relieved by double-J ureteral stent after ureteroscopy.

Nephrogenic diabetes insipidus is caused by the inability of the renal collecting ducts to absorb water in response to antidiuretic hormone (ADH), also known as vasopressin. Approximately $90 \%$ of patients are male with the X-linked recessive form, which is caused by a defect in the vasopressin V2 receptor in renal collecting duct cells. The remaining $10 \%$ of patients have autosomal NDI, which is caused by mutations in the gene encoding the aquaporin-2 water channel on chromosome $12 \mathrm{q} 13 .{ }^{1}$ As a result, patients with this disorder are not likely to have a good response to hormone replacement (such as desmopressin acetate [DDAVP]) or to drugs that increase either the renal response to $\mathrm{ADH}$ or $\mathrm{ADH}$ secretion. Thiazide diuretics in combination with a low solute diet can diminish the degree of polyuria in patients with NDI. The potassium-sparing diuretic amiloride also may be helpful due to its additive effect with the thiazide diuretic.

Nephrogenic diabetes insipidus is an uncommon cause of severe nonobstructive urinary tract dilatation. ${ }^{2} \mathrm{~A}$ review of the literature revealed that dilatation of the urinary tract was present in $67 \%$ of the reported cases of NDI. ${ }^{3}$ Rarely, there may be progressive
Corresponding Author:

Naishi Li, MD

Key Laboratory of Endocrinology of the Ministry of Health

Department of Endocrinology

Peking Union Medical College Hospital

Shuai Fu Yuan I\#, DongCheng District

Beijing, 100730, China

Tel: 86-10-65295073

Fax: 86-10-65295872

Email: laserpumc@gmail.com
Keywords: Hydronephrosis, Nephrogenic diabetes insipidus, Vasopressin

Received: May 3, 2009

Revised: July 21, 2009

Accepted: July 29, 2009

doi: $|0.3| 2 \mid / \mathrm{cmr} .2009 .860$

The Aperture, like the opening in the lens of a microscope that allows light to pass through, is a forum for art, humor, and images that provides a portal for new or different views of medicine and research. 


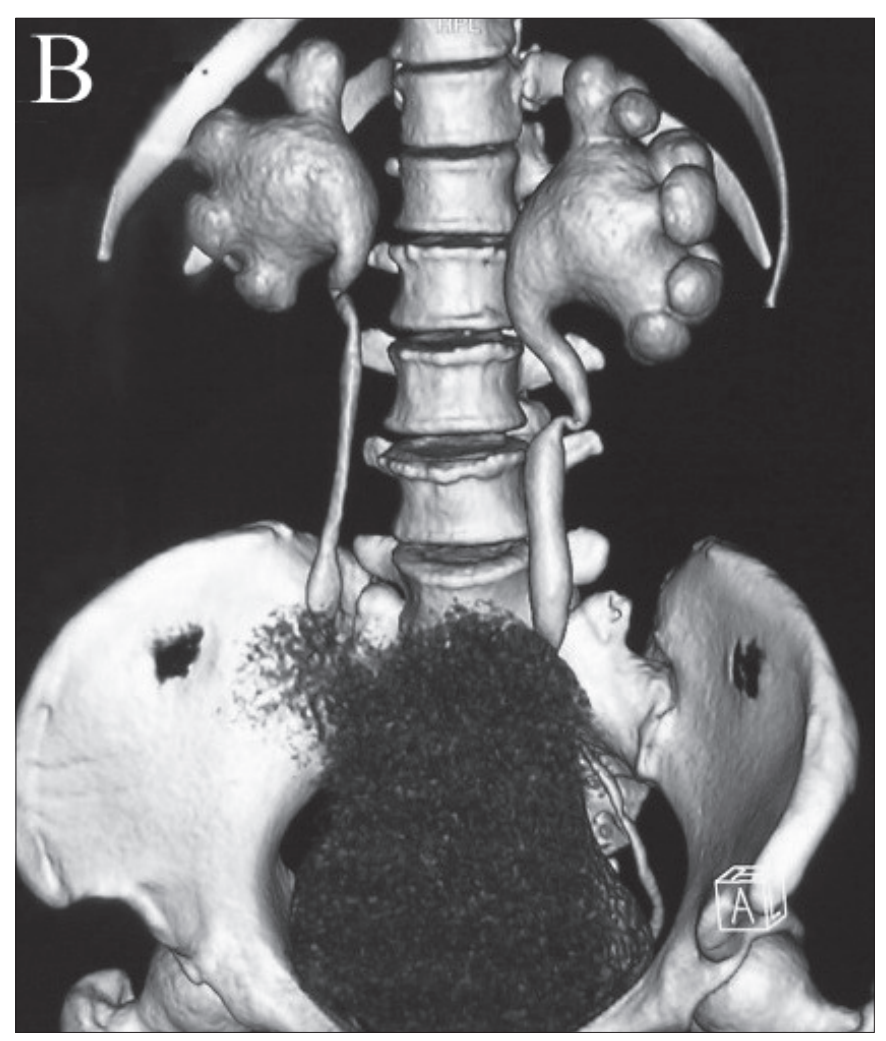

Figure 1B. Three-dimensional computed tomography of the kidneys.

loss of renal function and end-stage renal disease, probably related to voluntary retention of urine leading to bladder dysfunction. Drainage procedure is necessary to prevent severe dilatation of the urinary tract due to ongoing polyuria.

\section{References}

1. Morello JP, Bichet DG. Nephrogenic diabetes insipidus. Annu Rev Physiol 2001;63:607-630.

2. Stevens S, Brown BD, McGahan JP. Nephrogenic diabetes insipidus: a cause of severe nonobstructive urinary tract dilatation. J Ultrasound Med 1995;14:543-545.

3. Uribarri J, Kaskas M. Hereditary nephrogenic diabetes insipidus and bilateral nonobstructive hydronephrosis. Nephron 1993;65:346-349.

\section{Author Affiliations}

Hongbo Yang, MD, PhD; Naishi Li, MD;

Lingling Xu, MD; Weibo Xia, MD;

Key Laboratory of Endocrinology of the Ministry of Health, Department of Endocrinology,

Peking Union Medical College Hospital,

Shuai Fu Yuan 1\#, DongCheng District,

Beijing, 100730, China 